\title{
REDETERMINATION OF THE ORBIT OF IAPETUS
}

\author{
SHEN KAIXIAN AND QIAO RONGCHUAN \\ United Laboratory for Optical Astronomy, \\ Chinese Academy of Sciences, Shaanxi Observatory, \\ P.O.Box 18, Lintong, Shaanxi, China
}

Abstract. This paper gives a test of the reliability of TASS from Vienne \& Duriez via the comparison of the theory of Iapetus given by Harper \& Taylor. From fitting it to photographic observations, we derived a redetermination of the orbit of Iapetus

A new general theory of the motion of the eight satellites of Saturn - TASS (Théorie Analytique des Satellites de Saturne), has been built by Vienne \& Duriez 1991, 1992). Harper \& Taylor (1993) have completed also a new determination of the orbits of the major satellites of Saturn. In their paper a set of new analytical formulae of the perturbation for Iapetus was given.

The intention of our work is to construct a feasible analytical representation of the perturbed motion of Iapetus, based on TASS. But this paper only gives a test of the reliability of TASS 1.6 (Vienne \& Duriez, 1995) via a comparison with the theory of Iapetus given by Harper \& Taylor. We selected only those terms greater than $50 \mathrm{~km}$, which are derived from TASS 1.6 and should be compatible in precision with the terms obtained by Harper \& Taylor. In our computations, we have used the same variables used in TASS: $p, q, z, \zeta$ and the following values: $\dot{\omega}=\dot{\phi}=0 .{ }^{\circ} 1131$, $\dot{\Omega}=\dot{\Phi}=-0 .{ }^{\circ} 1103, \dot{\omega}_{T}=\dot{\phi}_{T}=0 .{ }^{\circ} 5118, \dot{\Phi}_{T}=-0 .{ }^{\circ} 5117$. We easily obtain the expression for the short perturbations of Iapetus. For example from $\Delta z=\Delta h+i \Delta k$, we may deduce $\delta e=\Delta h \cos \omega+\Delta k \sin \omega$, so

$$
\begin{aligned}
10^{5} \delta e= & 59.38 \cos \left(\ell_{T}+\omega_{T}-\omega-63 .{ }^{\circ} 12\right) \\
& +27.39 \cos \left(2 \ell_{T}-\ell_{T}+\omega-\omega_{T}+63 .^{\circ} 12\right) \\
& +25.33 \cos \ell+10.49 \cos \left(-\ell+2 \ell_{S}-2 \omega-188 .{ }^{\circ} 25\right) \\
& +2.06 \cos \left(3 \ell_{S}-\ell-2 \omega+160 .{ }^{\circ} 78\right) \\
& +4.89 \cos \left(3 \ell_{S}-2 \omega-261 . .^{\circ} 33\right) \\
& +37.89 \cos \left(2 \ell_{S}-2 \omega+187 . .^{\circ} 53\right) \\
& +1.72 \cos \left(2 \ell_{T}+\omega_{T}-\omega+112 . .^{\circ} 29\right) .
\end{aligned}
$$


in which $\ell, \ell_{T}, \ell_{S}$ have the same value as used in $\mathrm{H} \& \mathrm{~T}$, because they are just the difference between the longitudes and do not depend on the adopted reference frame. In computing long-period, resonant and secular terms, we use all those terms of TASS 1.6 given by Vienne and Duriez (1995; tables $8 \mathrm{a}, 8 \mathrm{~b}, 8 \mathrm{c})$.

The epoch and reference frame adopted by us are the same used by Harper \& Taylor. The starting epoch is 1930 January $24^{d} 0^{h}$ Ephemeris Time (JED-2426000.5). The mean ecliptic and equinox of B1950.0 was used as the fixed reference system. This is related to two reasons: (1) It will be convenient for comparison of our results with those of Harper \& Taylor; (2) We still can use the consistent partial derivatives with them. But, in TASS, the reference plane was assumed to be the equatorial plane of Saturn. The longitudes of node and pericenter are measured in the equatorial plane of Saturn. The origin of the longitudes is the node of this plane on the mean ecliptic J2000 frame. Therefore, it remains to do the rotations corresponding to the inclinations and the longitude of node of the equatorial plane in this frame (i.e., respectively, $28 .^{\circ} 0752$ and $169 .^{\circ} 5084$ ). Finally, the conversion from the J2000.0 reference system to B1950 was done with the aid of Lieske's (1994) formulae. In fact, this small adjustment is insignificant. As a test, we have fitted the two theories to 1763 photographic observations from 1967 to 1988 . The obtained r.m.s. residuals are $0^{\prime \prime} .2213$ for TASS and $0^{\prime \prime} .2215$ for H\&T. The preliminary results seem to indicate that it will be possible for the new theory to lead to some small improvements in precision in the determination of the orbit of Iapetus. Table I gives a set of new elements of the orbit of Iapetus corresponding to the date JED 2426000.5. In effect, no distinct differences between the two sets were found. The more refined part of this work is still in progress.

TABLE 1. Orbital elements of Iapetus

\begin{tabular}{ccccccc}
\hline $\begin{array}{c}\text { Elements } \\
\text { Units }\end{array}$ & $\begin{array}{c}a_{0} \\
\text { A.U. }\end{array}$ & $\begin{array}{c}\lambda_{0} \\
\text { deg. }\end{array}$ & $e_{0}$ & $\begin{array}{c}\varpi_{0} \\
\text { deg. }\end{array}$ & $\begin{array}{c}i_{0} \\
\text { deg. }\end{array}$ & $\begin{array}{c}\Omega_{0} \\
\text { deg. }\end{array}$ \\
\hline H\& T & 0.0238117 & 216.99743 & 0.0288367 & 357.824 & 18.02066 & 141.475 \\
S\& Q & 0.0238099 & 216.99519 & 0.0290824 & 357.856 & 18.02032 & 141.457 \\
\hline
\end{tabular}

\section{References}

Duriez, L. and Vienne, A.: 1991, Astron. Astrophys. 243, 263.

Harper, D. and Taylor, D.: 1990, Astrophys. 268, 326. (H\& T)

Lieske, J.H.: 1994, Astron. Astrophys. 281, 281.

Vienne, A. and Duriez, L.: 1991, Astron. Astrophys. 246, 619.

Vienne, A. and Duriez, L.: 1992, Astron. Astrophys. 257, 331.

Vienne, A. and Duriez, L.: 1995 Astron. Astrophys. 297, 588. 